\section{Zur Zersetzung anorganischer Kristalle im Elektronenmikroskop}

Von Ludwig Reimer

Physikalisches Institut der Universität Münster (Westf.) (Z. Naturforschg. 14 a, 759-760 [1959]; eingegangen am 18. Juli 1959)

Bei der Beobachtung von undurchstrahlbaren Kristallen im Elektronenmikroskop ist mit einer sehr starken Temperaturerhöhung zu rechnen, die bei einer kritischen Bestrahlungsintensität (in $\mathrm{A} / \mathrm{cm}^{2}$ ) zum Verdampfen und Zersetzen des Objektes führen kann. An Salzkristallen liegen hierüber zahlreiche Untersuchungen vor (s. u. a. Bills und Lefker ${ }^{1}$, Fischer ${ }^{2}$, Kinder ${ }^{3}$, König ${ }^{4}, \mathrm{McLlenNan}^{5}, \mathrm{~S}_{\mathrm{Awhill}}{ }^{6}, \mathrm{~T}_{\text {albot }}{ }^{7}$ oder $\mathrm{W}_{\text {Atson }}{ }^{8}$ ).

In vielen Fällen (z. B. bei den Alkalihalogeniden) handelt es sich um eine reine Verdampfung, denn man findet nach intensivem Elektronenbeschuß der Kristalle die Substanz teilweise als Aufdampfschicht in der Nähe der ursprünglichen Kristalle auf der Trägerfolie wieder. Vom Ausgangskristall pflegt nur eine dünne Kohlehülle übrig zu bleiben, die von den zu Anfang des Elektronenbeschusses niedergeschlagenen Kohleschichten herrührt. Andere Kristalle (insbesondere die Silberhalogenide) erleiden jedoch sicher eine Zersetzung.

Es liegen bisher noch keine quantitativen Messungen der Bestrahlungsintensität vor, bei der die Verdampfung einsetzt. Eine Kenntnis dieser Größe ist für die elektronenmikroskopische Praxis von Bedeutung, um einen Anhaltspunkt zu gewinnen, bis zu welchen Bestrahlungsintensitäten man bei der Untersuchung kristalliner Pulver gehen darf, ohne Objektveränderungen zu erhalten, oder bei welchen Intensitäten anorganische Schmutzteilchen verdampfen. Außerdem steht noch die Frage offen, ob zu der beobachteten Verdampfung der Kristalle im Elektronenmikroskop eine Erhöhung der Temperatur bis zur Schmelztemperatur erforderlich ist, oder ob nicht durch den Elektronenbeschuß eventuell eine Bindungslockerung erfolgt, die ein vorzeitiges Schmelzen und Verdampfen begünstigt.

Bei Folien (Trägerfolien, Abdruckfilmen oder Dünnschnitten) ist nach neueren Untersuchungen (Zusammenfassung s. Anm. ${ }^{9}, 10$ ) die Wärmeabgabe nur durch Wärmeleitung bedingt, wenn die Blendenöffnung nicht zu groß ist. Bei dickeren Schmutzteilchen oder undurchstrahlbaren Kristallen (Größenordnung $1 \mu$ ) ist dagegen mit vorwiegender Wärmeabgabe durch Strahlung zu rechnen.

Die vom Elektronenstrahl pro sec an den Kristall abgegebene Energie $Q_{\mathrm{E}}$, welche die Erwärmung verursacht, berechnet sich aus der Stromdichte $i$ inA $/ \mathrm{cm}^{2}$, der mittleren Energieabgabe $\Delta U$ eines Elektrons in Volt und

\footnotetext{
1 A. E. Bills u. R. Lefker, J. Appl. Phys. 25, 901 [1954].

2 R. B. Fischer, J. Appl. Phys. 25, 894 [1954].

3 E. Kinder, Naturwiss. 34, 23 [1947].

${ }^{4}$ H. König, Naturwiss. 35, 261 [1948].

5 D. E. McLennan, Canad. J. Phys. 29, 122 [1951].

6 J. Sawkill, Proc. Roy. Soc., Lond. A 229, 135 [1955].

7 J. H. Talbot, Brit. J. Appl. Phys. 7, 110 [1956].
}

der dem Elektronenstrahl entgegenstehenden Fläche $F$ $\mathrm{zu}$

$$
Q_{\mathrm{E}}=i \Delta U F .
$$

Bei einer Temperatur $T$ des Kristalls ( $T_{0}$ absolute Temperatur der Umgebung) beträgt die pro sec abgestrahlte Energie $Q_{\text {Str }}$ nach dem Stefan-Boltzmannschen Strahlungsgesetz

$$
Q_{\mathrm{Str}}=\sigma A O\left(T^{4}-T_{0}{ }^{4}\right) .
$$

Hierin bedeuten $\sigma=5,76 \cdot 10^{-12}$ Watt $\mathrm{cm}^{-2} \operatorname{grad}^{-4} \mathrm{die}$ Strahlungskonstante, $A$ das Absorptionsvermögen (=1 für den schwarzen Strahler) und $O$ die Oberfläche des Kristalls.

Im Gleichgewicht $\mathrm{mu} \beta Q_{\mathrm{E}}=Q_{\mathrm{Str}}$ sein und bei Erreichung der Schmelztemperatur $T_{\mathrm{s}}$ erhält man aus (1) und (2) für die kritische Stromdichte $i_{\text {krit }}$

$$
i_{\text {krit }}=\frac{\sigma A}{\Delta U} \frac{O}{F}\left(T_{\mathrm{s}}{ }^{4}-T_{0}{ }^{4}\right) .
$$

Für die folgenden Betrachtungen kann man ohne weiteres $T_{0}{ }^{4}$ gegenüber $T_{\mathrm{s}}{ }^{4}$ vernachlässigen. Das Verhältnis $O / F$ beträgt z. B. bei einem Würfel 6 , bei einer Kugel 4 . Es wäre also zu erwarten, daß die kritische Stromdichte proportional zur 4. Potenz der Schmelztemperatur ansteigt, wenn die Wärmeabgabe vorwiegend durch Strahlung erfolgt und die Wärmeleitung durch die Trägerfolie demgegenüber zu vernachlässigen ist.

Zur Ermittlung von $i_{\text {krit }}$ wurde die Bestrahlungsintensität bei $60 \mathrm{kV}$ Beschleunigungsspannung langsam so weit gesteigert, bis das Verdampfen der Kristallite einsetzte. Bei Erreichung des Schmelzpunktes ist in der Regel der Dampfdruck so hoch, da $\beta$ die Kristallite auch sehr schnell verdampfen. Die Messung von $i_{\text {krit }}$ erfolgte in der Bildebene mit einem FARADAY-Käfig und Elektrometer (Meßanordnung s. Anm. ${ }^{11}$ ) und Umrechnung auf die Objektebene durch Multiplikation mit dem Quadrat der Bildvergrößerung $(V=8000$-fach $)$. Die Kristallite hatten Abmessungen von 0,5 bis $1 \mu$ Durchmesser und wurden durch Eintrocknen von Suspensionen oder Lösungen auf Formvarfolien erhalten. Die Meßergebnisse sind in doppeltlogarithmischer Darstellung in Abb. 1 aufgetragen. Auf Grund von (3) ist dann zu erwarten, daß sich die Ergebnisse durch eine Gerade mit der Steigung 4 (ausgezogene Gerade) annähern lassen. Hiermit ist das Überwiegen des Wärmeverlustes durch Abstrahlung experimentell bestätigt.

Für quantitative Abschätzungen (es interessiert insbesondere der Wert von $\Delta U$ ) kann man als mittleren Wert $O / F=5$ ansetzen. Da das Emissionsvermögen eines Stoffes nach dem KirchHofrschen Gesetz mit dem Absorptionsvermögen zusammenhängt und die Salzkristalle zum Teil bis weit in das Ultrarote durchlässig sind, ist

8 J. H. L. Watson, J. Appl. Phys. 19, 713 [1948].

9 S. Leisegang, Elektronenmikroskope, in: Handbuch der Physik Bd. 33 [1956].

10 L. ReImer, Elektronenmikroskopische Untersuchungs- und Präparationsmethoden, Springer-Verlag, Berlin-GöttingenHeidelberg 1959.

11 L. Reimer, Z. Naturforschg. 14 b, in Vorbereitung. 


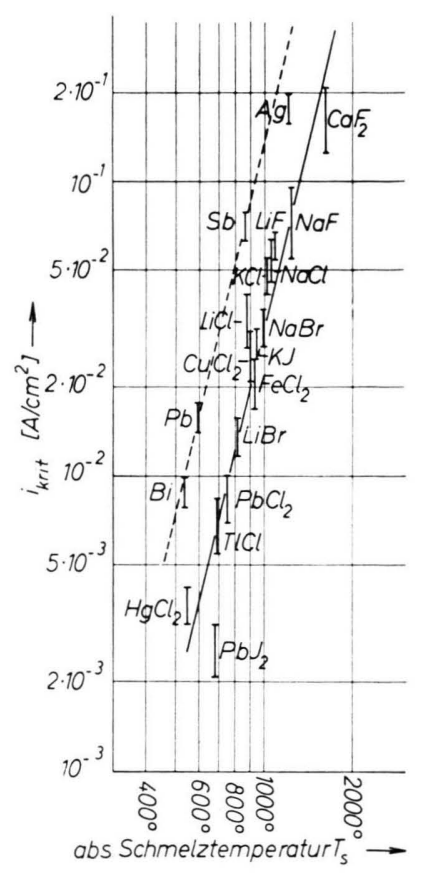

Abb. 1. Abhängigkeit der kritischen, zum Verdampfen von Salzen und Metallen erforderlichen Stromdichte $i_{\text {krit }}$ bei $60 \mathrm{kV}$ Strahlspannung in der Objektebene von der Schmelztemperatur $T_{\mathrm{s}}$ in doppeltlogarithmischer Darstellung.

$A$ auf jeden Fall bedeutend kleiner als 1 (graue Strahler). Genauere Messungen liegen nur an Metallen vor ${ }^{12}$. Die Abstrahlung beträgt demnach nur etwa $2-3 \%$ der

\section{Zur Dipolrelaxation von Fehlstellenkomplexen in Silberhalogeniden mit Zusätzen}

\section{Von Manfred Höhne}

Institut für Kristallphysik

der Deutschen Akademie der Wissenschaften zu Berlin

(Z. Naturforschg. 14 a, 760-762 [1959]; eingegangen am 29. Juni 1959)

Ziel der vorliegenden Untersuchungen war eine weitere Klärung der Zentrenvorstellungen in Silberhalogeniden. Eine aufschlußreiche Methode zur Untersuchung von Fehlordnungserscheinungen in Ionenkristallen ist die Messung des dielektrischen Verlustfaktors $\operatorname{tg} \delta$. In früheren Untersuchungen an Silberhalogeniden mit $\mathrm{Zu}$ sätzen hatten Teltow und $\mathrm{W}_{\mathrm{ILKE}}{ }^{1}$ sowie Busse und Teltow ${ }^{2}$ Maxima in der Temperaturabhängigkeit des Verlustfaktors gefunden und diese Maxima einfachen Fehlstellendipolen zugeordnet. Durch Verbesserung der Versuchstechnik und Erweiterung des Temperaturbereiches wurde eine Anzahl neuer Maxima entdeckt und Aufschluß über Zusammenhang und Veränderung der zugehörigen Zentren gewonnen.

1 J. Teltow u. G. Wilke, Naturwiss. 41, 423 [1954].

2 J. Busse u. J. Teltow, Naturwiss. 44, 111 [1957]. abgestrahlten Energie, die ein schwarzer Körper gleicher Temperatur emittieren würde. Außerdem ist zu vermerken, daß Abweichungen vom $T^{4}$-Gesetz (2) in dem Sinne auftreten können, daß der Exponent etwas größer als 4 ausfällt. Die Streuungen der Meßwerte können also sowohl auf unterschiedliche Werte von $A$ der einzelnen Salze als auch auf Abweichungen vom $T^{4}$-Gesetz zurückzuführen sein. Deshalb wurden die gleichen Versuche an einigen Metallpulvern durchgeführt, die ebenfalls in Abb. 1 eingetragen sind. Aus der gestrichelten Geraden ergibt sich $A / \Delta U=4 \cdot 10^{-3}$. Mit obigen Werten $(A=2-3 \%)$ ergibt sich als grobe Abschätzung $\Delta U=5-8 \mathrm{~V}$. Gemessen an der gesamten Beschleunigungsspannung von $60 \mathrm{kV}$ ist dieser Betrag, der als Energie in Elektronenvolt im Kristall stecken bleibt und zur Erwärmung beiträgt, sehr gering. Man muß aber bedenken, daß sehr viele Elektronen im Kristall nur elastische Mehrfachstreuung erleiden und den Kristall wieder ohne Energieverlust verlassen können. (Die Richtungsänderungen sind dabei so groß, da $\beta$ bei den geringen Beobachtungsaperturen der Kristall als undurchstrahlbar erscheint.) Bei dünnen durchstrahlbaren Schichten rechnet man mit einem mittleren Energieverlust von 0,2 bis $0,5 \mathrm{eV}$ pro Elektron ${ }^{9}$. Der in den hier vorliegenden Kristallen von 0,5 bis $1 \mu$ Dicke abgeschätzte Energieverlust von 5 bis $8 \mathrm{eV}$ liegt also durchaus in der richtigen Größenordnung.

In weiteren Versuchen soll geklärt werden, wieweit die Zersetzung der Silberhalogenide ein reiner DosisEffekt ist. Die Versuche wurden mit einem Siemens-Elmiskop I durchgeführt, welches der Universität Münster von der Deutschen Forschungsgemein $\mathrm{sch}$ a f $\mathrm{t}$ zur Verfügung gestellt wurde.

12 E. Hagen u. H. Rubens, Ann. Phys., Lpz. 11, 873 [1903].

Es wurden zunächst Messungen an $\mathrm{AgBr}$ mit zweiwertigen Anionenzusätzen $\left(\mathrm{Ag}_{2} \mathrm{Se}, \mathrm{Ag}_{2} \mathrm{~S}\right.$, ferner $\mathrm{Ag}_{2} \mathrm{Te}$, $\mathrm{Ag}_{2} \mathrm{O}$ ) in einem Bereich der Frequenz $v$ von $200 \mathrm{~Hz}$ bis $10 \mathrm{kHz}$ durchgeführt. Aus der Verschiebung der Maxima bei Änderung der Frequenz wurde u. a. jeweils die Schwellenenergie für die Orientierungsänderung der Fehlstellendipole bestimmt.

Da die vorliegenden Untersuchungen an polykristallinem Material (Korndurchmesser ca. $1 \mathrm{~mm}$ ) durchgeführt wurden, war die Abschätzung des Einflusses von Baufehlern notwendig. Orientierende Messungen an getemperten Einkristallen einerseits und an kalt verformten Polykristallen andererseits ließen nur triviale Einflüsse erkennen, wie etwa eine Erhöhung der Grundkurve in versetzungsreichem Material auf Grund der höheren Leitfähigkeit.

Kurve $I$ in Abb. 1 zeigt die Abhängigkeit des $\operatorname{tg} \delta$ von der Temperatur $T$ bei $\nu=800 \mathrm{~Hz}$ für $\mathrm{AgBr}$ mit $0,04 \%$ (Mol-\%) $\mathrm{Ag}_{2} \mathrm{Se}$ für einen Kristall, der von einer Temperatur knapp unter dem Schmelzpunkt rasch auf $20{ }^{\circ} \mathrm{K}$ abgekühlt wurde. Besondere Aufmerksamkeit sei in dieser Notiz dem Maximum (A) bei $44{ }^{\circ} \mathrm{K}$, das den Komplexen Fremdion-Silberion auf Zwischengitterplatz zugeordnet wurde, und dem Maximum (B) bei $106{ }^{\circ} \mathrm{K}$ gewidmet. Ein dem Maximum A entsprechendes 